\section{Aplicação de métodos de estimativa da prevalência de hanseníase no Estado de Mato Grosso}

\section{Application of estimation methods for the hidden prevalence of leprosy in the State of Mato Grosso}

\section{Eliane Ignotti}

Departamento de Ciências Biológicas

Universidade do Estado de Mato Grosso (UNEMAT/FIOCRUZ)

Campus Universitário de Alta Floresta - MT

Rod. MT208, km 147/146

eignotti@ensp.fiocruz.br

\section{Alex Miranda Rodrigues}

Departamento de Medicina Comunitária

Faculdade de Medicina

Universidade de Cuiabá (UNIC)

Vera Lúcia Gomes de Andrade

Organização Pan-americana de Saúde (OPAS/OMS)

\section{Joaquim Gonçalves Valente}

Departamento de Epidemiologia e Métodos Quantitativos

Escola Nacional de Saúde Pública (ENSP/FIOCRUZ)

Instituto de Medicina Social

Universidade Estadual do Rio de Janeiro (IMS/UERJ)

\section{Resumo}

Uma questão desafiadora para a eliminação da hanseníase como problema de saúde pública é o conhecimento da sua real prevalência. Este estudo tem por objetivo comparar os resultados de dois métodos publicados como propostas de estimativa de prevalência oculta de hanseníase, a tendência definida pelo Ministério da Saúde, e a estimativa realizada pela Secretaria de Estado de Saúde de Mato Grosso (SES/MT) para a implantação do programa "Tolerância Zero: Mato Grosso sem hanseníase”, tendo como parâmetro o número de casos novos detectados em 2001.

Fez-se a aplicação dos métodos e da tendência, utilizando-se a série de casos de hanseníase detectados no período de 1996 a 2000 para todos os municípios do Estado. Definiu-se como nível endêmico a média aritmética ajustada aos casos novos detectados no mesmo período. Verificou-se que todas as estimativas mostraram associação positiva maior que $75 \%$ com o número de casos detectados em 2001. A maior associação foi verificada entre a meta ou estimativa da SES/MT $\left(r^{2}=0,895\right)$, que por outro lado não é observada com a exclusão do nível endêmico. Concluímos que os métodos publicados apenas sugerem a presença de reservatórios de casos em áreas com detecção tardia. Operacionalmente, parece mais adequado basear-se em séries históricas segundo idade, classificação operacional, grau de incapacidade física e número de lesões no momento do diagnóstico para a identificação de áreas de risco e, conseqüentemente, eliminação da hanseníase.

Palavras-chave: Hanseníase. Estimativa de prevalência. Mato Grosso. 


\section{Introdução}

One of the challenges to eliminate leprosy as a public health problem is to know its real prevalence. This study compares the results of two published methods which propose to estimate the hidden prevalence of leprosy, the trends defined by the Ministry of Health and the estimate made by the State Secretary of Health of Mato Grosso (SES/ MT) to implement the program "Tolerância Zero: Mato Grosso sem hanseníase” (Zero Tolerance: Mato Grosso Without Leprosy), based on the number of new cases detected in 2001 .

The methods and the trend were applied using the cases of leprosy detected in the period from 1996 to 2000 for all the municipalities of the state. The endemic level was defined as the arithmetic mean adjusted to the new cases detected in the same period. All estimates showed a positive association higher than $75 \%$ with the number of cases detected in 2001. The strongest association was obtained between the estimate of SES/ MT $\left(r^{2}=0,895\right)$, which, in contrast, does not exclude the endemic level. We conclude that the published methods only suggest the presence of reservoirs of cases in areas with delayed detection. Operationally, it seems more adequate to rely on historical series according to age, operational classification, degree of physical incapacitation, and number of lesions at the time of diagnosis to identify the areas of risk and, consequently, eliminate leprosy.

Key Words: Leprosy. Prevalence estimate. Mato Grosso.
O quadro epidemiológico de Mato Grosso revela elevada prevalência de hanseníase, alcançando valores acima de 24 casos por 10.000 habitantes. Na busca da eliminação da hanseníase como problema de saúde pública até 2005, a Secretaria de Estado de Saúde (SESMT) implantou o "Projeto Prioritário Tolerância Zero: Mato Grosso sem hanseníase" em junho de $2001^{1}$. Tendo em vista a execução do projeto, foi realizada uma estimativa de casos novos de hanseníase, e subseqüentemente da prevalência definida enquanto meta, para todos os municípios do Estado. Contudo, uma questão desafiadora é o conhecimento da real prevalência da endemia, indicador que vem sendo desenvolvido por meio de estimativas. Assume-se que a prevalência real da hanseníase resulta da soma da prevalência conhecida com a prevalência oculta a ser estimada. Estas estimativas respaldariam a elaboração de estratégias para a detecção de casos, identificação de focos de transmissão e conseqüente eliminação da doença.

O objetivo deste estudo é comparar os resultados de dois métodos publicados como propostas de estimativa de prevalência oculta de hanseníase, a tendência proposta pelo Ministério da Saúde e a estimativa realizada pela SES/MT, tendo como parâmetro os resultados do projeto “Tolerância Zero" para o ano 2001.

\section{Projeto prioritário Tolerância Zero: estimativa e custos}

Uma característica do projeto foi a definição de bônus para os Agentes Comunitários de Saúde por cada caso novo suspeito que tenha o diagnóstico confirmado, outro bônus a ser dividido pela equipe de saúde responsável pelo caso curado, e prêmios aos municípios que atingirem as metas estabelecidas referentes à detecção, cura e prevalência. Para a definição do número de casos referentes às metas, foi estimado o número de casos novos para cada município do Estado, para o ano de 2001, e assim se fez a previsão orçamentária do projeto. 
A SES-MT agrupou os municípios em quatro estratos, tendo como parâmetros o número de habitantes seguido da prevalência, apresentados como níveis de prioridade.

- Prioridade I - Municípios com população acima de 20.000 habitantes e prevalência acima de 20 casos por 10.000 habitantes;

- Prioridade II - Municípios com população acima de 20.000 habitantes e prevalência maior que 15 casos por 10.000 habitantes;

- Prioridade III-Municípios com população acima de 15.000 habitantes e prevalência maior que 10 casos por 10.000 habitantes;

- Prioridade IV - Demais municípios do Estado.

A definição por estratos está vinculada aos valores dos prêmios (bônus) definidos para os municípios.

\section{Metodologia}

Propôs-se a aplicação de duas metodologias de estimativa da prevalência oculta de Hanseníase e da tendência proposta pelo Ministério da Saúde em comparação com a estimativa realizada pela SES-MT, para finalmente fazer a verificação dos resultados da intervenção do projeto “Tolerância Zero" por meio do número de casos detectados no ano de 2001. Foram utilizados os dados do SINAN (Sistema Nacional de Agravos de Notificação), fornecido pela Secretaria de Estado de Saúde em julho de 2002 e do DATASUS (Base de dados do Sistema Único de Saúde). Foram selecionados os registros de casos novos de hanseníase a partir de 1996 por local de residência, segundo grau de incapacidade física para todos os municípios do Estado. Além disso, utilizou-se, como variáveis de análise, os estratos de prioridade definidos pela SES-MT, Escritório Regional de Saúde de Vinculação dos municípios (ERSA), número de habitantes residentes no município e proporção de habitantes por unidade de saúde. Foram excluídos do estudo 17 municípios, que, somados, possuíam 54.013 habitantes ou 2,1\% da população do Estado de Mato Grosso. A exclusão destas localidades deveu-se ao fato de que, em pelo menos um dos métodos utilizados, o resultado da estimativa tenha sido igual a zero, o que aumentaria a variabilidade para a análise de associação na comparabilidade entre os métodos.

Durante o período em estudo ocorreu a implantação do SINAN nos municípios; portanto, verificou-se ao longo da série grande instabilidade dos registros de casos. Para reduzir a instabilidade (variabilidade na detecção) foi utilizada a distribuição de Poisson $^{2}$, em que o critério estabelecido para obtenção do intervalo de confiança foi: média mais/menos 2 vezes a raiz quadrada da média. A partir do intervalo de valores aceitos como mínimo e máximo, foram encontrados valores reconhecidos como "válidos". Calculou-se a média dos valores válidos, $\mathrm{e}$ posteriormente os valores "não-válidos" foram substituídos ou estimados, gerando o que se denomina média estimada.

O método proposto por Gil Suárez e Lombardi ${ }^{3}$ baseia-se no pressuposto de que o diagnóstico de casos com incapacidades físicas indica detecção tardia e, portanto, a presença de casos não detectados numa determinada área. A estimativa da prevalência oculta mais a média de casos novos durante a rotina nos últimos 5 (cinco) anos será igual ao número de casos novos a serem detectados e a prevalência real estimada. (Prevalência Real Estimada $=$ prevalência conhecida + prevalência oculta). Para encontrar a prevalência oculta da hanseníase, os autores recomendam a divisão dos casos que apresentam incapacidades físicas pelo número de casos avaliados; assim é estimada a proporção de casos com incapacidades físicas. Posteriormente, aplica-se este percentual aos casos novos para se conhecer o número de casos que deveriam apresentar incapacidades se todos os casos conhecidos tivessem sido avaliados. Os outros métodos propõem que o número de casos estimados como tendo incapacidades seja praticamente igual ao número de casos ocultos a cada ano. Finalmente, soma-se os casos ocultos dos cinco 
anos da série, o que resulta no número de casos a ser detectado. O pressuposto das estimativas é que cada caso com incapacidade tenha gerado um caso oculto de hanseníase.

Além da distribuição de Poisson utilizada para redução da instabilidade dos casos notificados, aplicamos ao método ${ }^{3}$ a ponderação dos percentuais obtidos ao longo da série. Assim, a proporção de casos detectados com incapacidades físicas foi obtida por meio da média de casos com incapacidades e de casos avaliados nos últimos cinco anos. Posteriormente, esta proporção ponderada foi aplicada aos casos novos de cada ano da série e, como no modelo anterior, a somatória dos casos ocultos dos últimos cinco anos representa o total de casos ocultos, que, somados à média, compõem a prevalência real estimada.

O segundo método foi publicado por Ferreira et al. ${ }^{4}$, que além do grau de incapacidade física no momento do diagnóstico incluíram o tempo de início dos sintomas como um dos parâmetros para a estimativa de prevalência oculta.

\section{$\mathrm{EHP}=[\Sigma(\mathrm{NCg} \times \mathrm{MDg})] /$ CGE $\times \mathrm{PCP}$}

Onde:

- $\mathrm{EHP}=$ Estimativa de prevalência oculta.

- $\mathrm{NCg}=$ Média anual de casos novos detectados em cada categoria de incapacidade.

- $\mathrm{MDg}=$ Média de tempo, em anos, de atraso de diagnóstico em cada categoria de incapacidade.

- CGE = Proporção de casos novos detectados com avaliação do grau de incapacidade.

- $\quad \mathrm{PCP}=$ Proporção da população coberta pelo programa de hanseníase.

Como tempo médio de atraso de diagnóstico, utilizou-se 1,74 anos para os casos avaliados como graus de incapacidade $0 \mathrm{e}$, e 5,18 anos para os casos graus II e III (o grau II agrega os graus II e III na atual definição operacional), que correspondem aos tempos de atraso no diagnóstico apresentados para o Estado do Rio Grande do Sul no período de 1970 a $1991^{4}$.

Para a definição da proporção da população coberta pelo programa de hanseníase (PCP) utilizou-se a mesma proporção de casos avaliados quanto ao grau de incapacidade física no momento do diagnóstico, considerando que, no Estado de Mato Grosso, devido a dificuldades no acesso às unidades de saúde, não levamos em conta o número de unidades por habitantes como indicador de cobertura do programa, mesmo porque apenas $26 \%$ das unidades básicas de saúde notificaram algum caso de hanseníase no ano de 2001 .

O terceiro método propõe uma estimativa por meio da tendência de crescimento da endemia aplicada à prevalência do ano anterior. Neste caso, trata-se de uma recomendação operacional do Ministério da Saúde, encontrada no Guia para implantar/ implementar as atividades de controle da hanseníase nos planos estaduais e municipais de saúde ${ }^{5}$ com a utilização de $5 \%$ de tendência de crescimento ou, se esta for conhecida, o percentual de tendência de crescimento específico daquele Estado. Segundo o estudo de tendência realizado por Motta e Zuniga $^{6}$, a média nacional de tendência de aumento da detecção foi de $5 \%$ e a média no Estado de Mato Grosso foi de 8\%. Aplicouse a tendência de 5 e $8 \%$ para os casos detectados no ano 2000 ( $\mathrm{N}^{\circ}$ de casos esperados $=$ $\mathrm{n}^{\circ}$ de casos do ano anterior $+\%$ de tendência).

Quanto à estimativa da SES-MT, foi feita uma avaliação da tendência percentual aplicada e buscou-se associação com variáveis como: população residente, cobertura populacional por unidade de saúde, ERSA de vinculação do município e estratos de prioridade segundo o projeto "Tolerância Zero".

Reduziu-se o que foi definido como "nível endêmico" (média de casos detectados nos últimos cinco anos) da meta ou estimativa da SES-MT e do número de casos detectados em 2000, e calculou-se a proporção de alcance da meta para os municípios em estudo. Para averiguação da melhor associação 
entre o número de casos novos de hanseníase ao final de 2001 e as estimativas produzidas segundo os vários métodos apresentados, utilizou-se gráficos de dispersão com reta de tendência para regressão linear (Rsq).

Definimos como municípios que aumentaram a capacidade de detecção (sucesso) no ano de 2001 apenas aqueles que tiveram resultado positivo na meta da SES-MT sem nível endêmico e no número de casos novos detectados em 2001, descontado o nível endêmico.

\section{Resultados}

No Estado de Mato Grosso, a cobertura populacional na atenção ao doente de hanseníase está próxima de $100 \%$, segundo os critérios do Ministério da Saúde. Dos 122 municípios em estudo, 5 (cinco) não possuem unidades de saúde e população abaixo de 5.000 habitantes; em 109 municípios, há uma unidade de saúde para no máximo 5.000 habitantes, e 8 (oito) municípios possuem uma unidade para uma população entre 5.000 e 14.788 habitantes.
Na Tabela 1, apresentamos os resultados das equações $\left(R^{2}\right)$ entre as estimativas em estudo e o número de casos de hanseníase detectados em 2001. Os resultados estão dispostos em ordem decrescente, de acordo com a associação observada. Verificou-se que todas as estimativas mostraram associação positiva maior que $75 \%$ com o número de casos detectados em 2001. Entretanto, a maior associação foi verificada entre a meta ou estimativa da SES-MT e o número de casos novos de hanseníase detectados em 2001, com um ajuste de quase $90 \%\left(\mathrm{r}^{2}=0,895\right)$. Não foi observada diferença nas regressões das tendências de 5 e $8 \%$. A média estimada, assim como a metodologia proposta por Gil e Lombardi ${ }^{3}$, quando calculadas por meio da média dos casos apresentaram resultados mais ajustados em relação à média bruta e do mesmo método sem ponderação. $\mathrm{O}$ município de Cuiabá foi excluído das regressões por apresentar um contingente populacional muito maior que os outros municípios do Estado.

Na Tabela 2 observa-se diferença de associação entre os resultados das regressões

Tabela 1 - Resultados das Equações lineares segundo estimativas de prevalência oculta de hanseníase $(\mathrm{x})$ e de casos novos detectados nos municípios do estado de Mato Grosso em $2001(\mathrm{y})$.

Table 1 - Results of linear equations according to estimates of hidden prevalence of leprosy $(x)$ and new detected cases in municipalities the State of Mato Grosso, 2001 (y).

\begin{tabular}{ll}
\hline Estimativas (X) & Regressão \\
\hline Meta da SES - MT & $y=0,9303 x-0,6919$ \\
& $r 2=0,895$ \\
Tendência de 5\% & $y=0,8345 x-1,7927$ \\
(MS, 1999) & $r 2=0,8056$ \\
Tendência de 8\% & $y=0,8583 x-1,8439$ \\
MOTTA \& ZUNIGA (1990) & $r 2=0,8056$ \\
Média estimada (distribuição de Poisson) & $y=0,761 \mathrm{x}-1,3023$ \\
& $\mathrm{r} 2=0,8019$ \\
GIL \& LOMBARDI (1997) & $\mathrm{y}=1,2169 \mathrm{x}+0,1258$ \\
(ponderado pela média dos cinco anos) & $\mathrm{r} 2=0,7649$ \\
MÉDIA aritmética (5 anos) & $\mathrm{y}=0,717 \mathrm{x}-0,5801$ \\
& $\mathrm{r} 2=0,7633$ \\
GIL \& LOMBARDI (1997) & $\mathrm{y}=1,2958 \mathrm{x}+0,3291$ \\
Ferreira et .al. (2000) & $\mathrm{r} 2=0,7325$ \\
& $\mathrm{y}=0,3341 \mathrm{x}+9,1786$ \\
& $\mathrm{r} 2=0,5938$ \\
\hline
\end{tabular}


da meta da SES-MT e casos novos de hanseníase detectados em 2001, com e sem o nível endêmico. Pode-se verificar que, no segundo caso, a associação é reduzida em $57 \%$, passando de $\mathrm{r} 2=0,895$ para $\mathrm{r} 2=0,3221$.

Nas Figuras 1 e 2 pode-se visualizar as diferenças de associação entre a meta da SESMT e o número de casos de hanseníase detectados em 2001, com e sem nível endêmico. Quando excluída a média ajustada (nível endêmico), tanto dos casos novos de 2001 quanto da Meta-SES, não é verificada associ- ação entre estas variáveis, ou seja, o que foi estimado para 2001 foi quase totalmente alcançado enquanto número de casos detectados. Observa-se vários pontos negativos nos quadrantes da função de regressão. Dos 122 municípios em estudo, verificou-se que, para 45 (36,9\%), a Meta (estimativa da SES/MT) foi proposta abaixo do nível endêmico. Tais municípios concentram 543.769 habitantes $(21 \%$ da população do Estado).

$\mathrm{Na}$ avaliação da tendência na Meta da SES-MT verifica-se que houve uma variação

Tabela 2 - Resultado das equações lineares segundo a meta de detecção de casos de hanseníase da SES-MT ( $\mathrm{x}$ ) e de casos novos detectados nos municípios do estado de Mato Grosso em 2001 (y) com e sem o nível endêmico.

Table 2 - Results of linear equations according to leprosy detection goals for the State Secretary of Health $(x)$ and new detected cases in municipalities of the State of Mato Grosso, $2001(y)$ with and without endemic level.

\begin{tabular}{ll}
\hline Variáveis & Regressão \\
\hline (x)Meta da SES com o nível endêmico & $\mathrm{y}=0,9303 \mathrm{x}-0,6919$ \\
(y)Casos novos de 2001 com o nível endêmico & $\mathrm{r} 2=0,895$ \\
& $\mathrm{y}=0,4351 \mathrm{x}+1,8213$ \\
(x)Meta sem o nível endêmico & $\mathrm{r} 2=0,3221$ \\
\hline
\end{tabular}

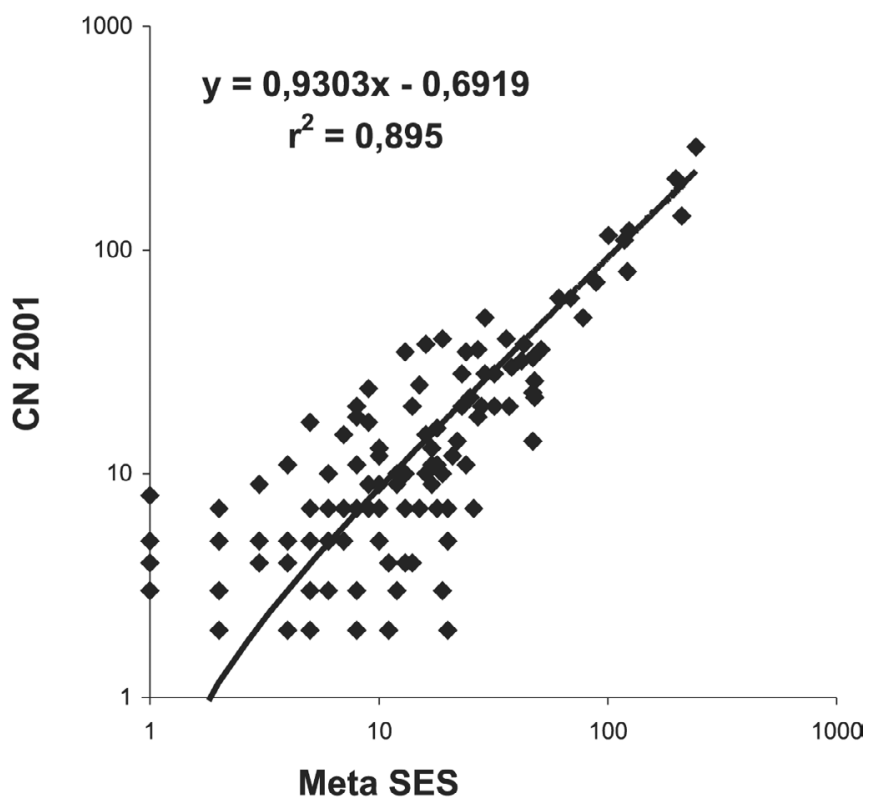

Figura 1 - Gráfico de dispersão dos casos detectados de hanseníase e dos casos estimados para detecção (Meta SES), nos municípios do estado de Mato Grosso, 2001.

Figure 1 - Scatter plotting of new detected cases of leprosy and cases estimated for detection (Goal SES), in municipalities of the State of Mato Grosso, 2001. 


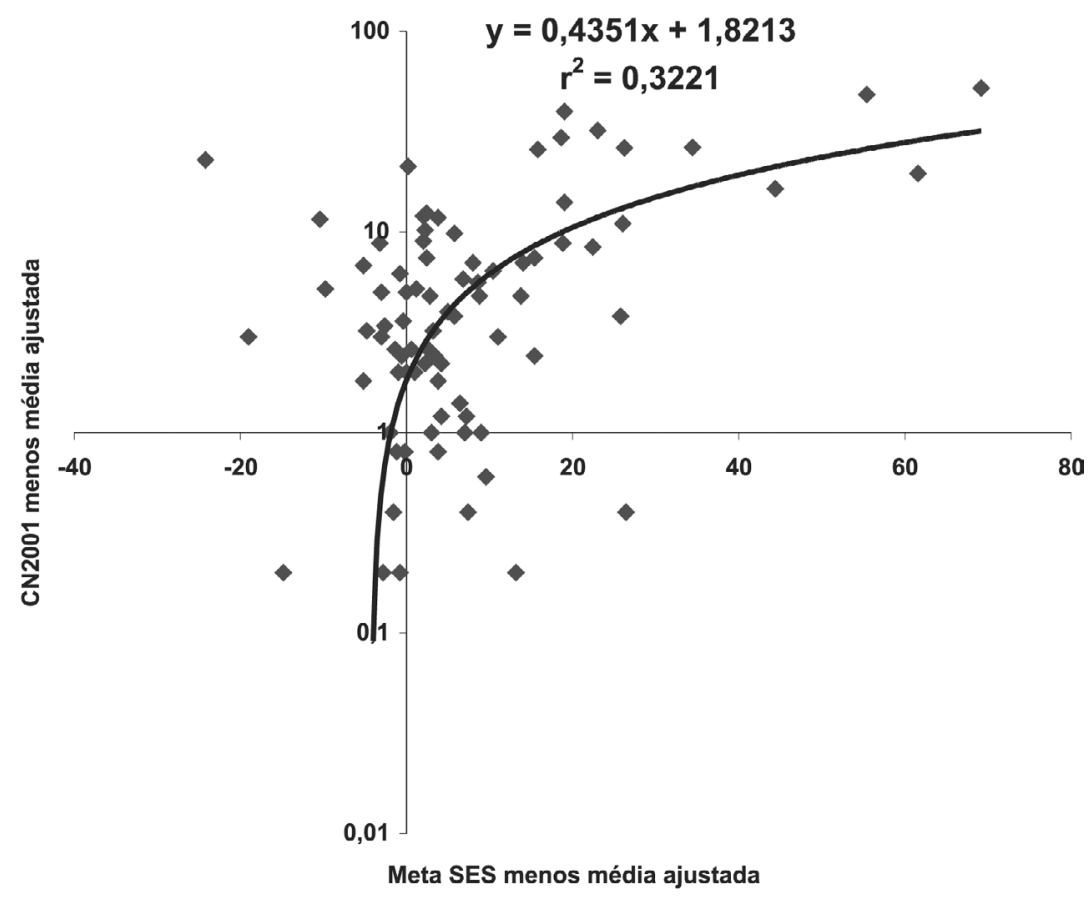

Figura 2 - Gráfico de dispersão dos casos detectados de hanseníase menos a média ajustada e dos casos estimados para detecção (Meta SES) menos a média ajustada, nos municípios do estado de Mato Grosso, 2001.

Figure 2 - Scatter plotting of new detected cases of leprosy minus adjusted average of cases estimated for detection (Goal SES) in municipalities of the State of Mato Grosso, 2001.

percentual de menos oitenta e seis $(-86,1 \%)$ até mil (1.000\%). A tendência negativa aplica-se aos 45 municípios em que a Meta foi menor que a média dos últimos 5 (cinco) anos, calculada com base nos casos detectados no ano 2000. Da análise dos resultados maiores que zero, ou seja, com alguma tendência de crescimento na detecção, nos 77 municípios verificou-se uma variação na tendência de 2,5\% a 1000\% (média $=138,4 \%$, mediana $=85 \%$ ). Não foi verificada associação da tendência da meta, ou seja, da estimativa de casos feita pela SES-MT, ainda que categorizada de acordo com os quartis, com nenhuma outra variável em estudo. Categorizando os municípios com a meta acima e abaixo da média, também não foi verificada associação com outras variáveis em estudo.

Na Figura 3 pode-se observar a distribuição dos 75 municípios com sucesso no aumento da capacidade de detecção em 2001, segundo a SES-MT, e na Figura 4 os 54 municípios que aumentaram a capacidade de detecção mesmo quando excluído o ní- vel endêmico. Não foi possível observar um padrão espacial dos municípios aqui considerados como bem sucedidos nas duas formas de análise e verifica-se ainda que, dos 54 municípios bem sucedidos com a exclusão do nível endêmico, apenas 37 estão de acordo com a avaliação da SES-MT.

\section{Discussão}

Alguns estudos foram realizados na produção de estimativas de prevalência oculta e outros na estimativa de tendência de ocorrência da hanseníase. Bechelli et. $\mathrm{al}^{7}$ propuseram um método para estimar os casos não detectados de hanseníase, baseado em dados do Grupo Consultor da Organização Mundial da Saúde que, se o programa de controle da endemia em dada região se revelava como satisfatório, regular ou precário, adiciona-se fator corretivo ao número de casos registrados, respectivamente de $75 \%, 150 \%$ e $300 \%$. Opromolla et al. ${ }^{8}$ realizaram estimativa da prevalência da hanseníase 


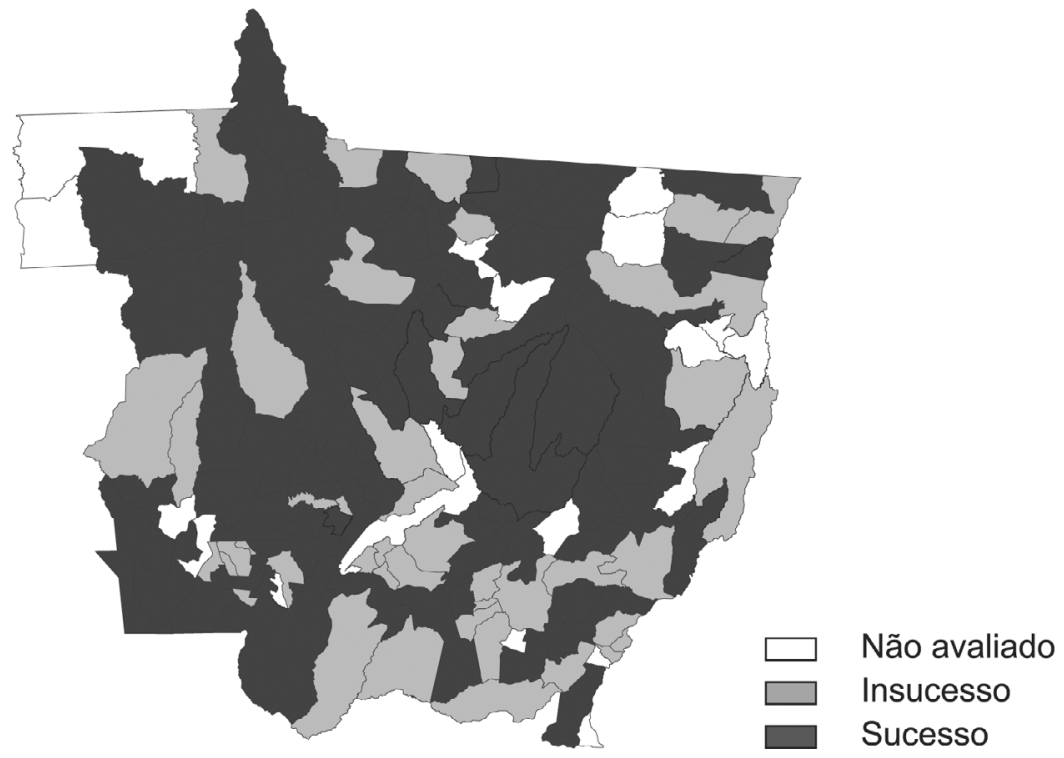

Fonte: SES-MT e SINAN - MT

Figura 3 - Municípios do estado de Mato Grosso segundo desempenho no aumento da capacidade de detecção de hanseníase no ano de 2001 incluindo o nível endêmico.

Figure 3 -Municipalities of the State of Mato Grosso according to their role in the increase of capacity to detect leprosy in the year of 2001 including endemic level.

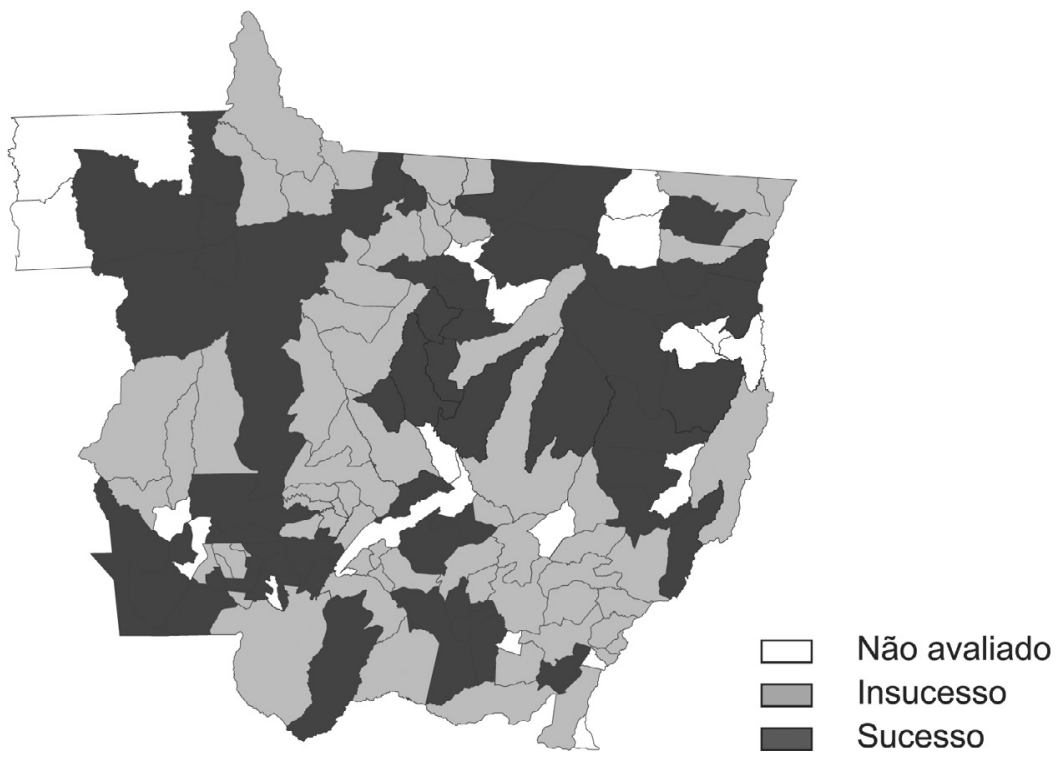

Fonte: SES-MT e SINAN - MT

Figura 4 - Municípios do estado de Mato Grosso segundo desempenho no aumento da capacidade de detecção de hanseníase no ano de 2001 mesmo com a exclusão do nível endêmico.

Figure 4 - Municipalities of the State of Mato Grosso according to their role in the increase of capacity to detect leprosy in the year of 2001, excluding endemic level. 
pela investigação em demanda inespecífica de unidades de saúde ${ }^{8}$. A principal discussão foi voltada para a busca da base do iceberg epidemiológico pelo qual se designa o fato de que o número de casos conhecidos da doença é sempre a extremidade visível de contingente muito mais numeroso. Foram utilizados exames clínicos e laboratoriais na população que buscou os serviços de saúde de Taubaté espontaneamente, por outras razões que não hanseníase, e verificou-se um acréscimo de um mínimo de $52 \%$ e máximo de $109 \%$ na prevalência, ou seja, valores muito distintos do fator corretivo proposto por Bechelli \& Martinez Dominguez.

Lechat ${ }^{9}$ refere-se à interrupção da transmissão para posterior eliminação da doença considerando-se a efetividade da poliquimioterapia (PQT) na redução dos reservatórios ou fontes de infecção dos pacientes com conseqüente eliminação da transmissão. Para ele, a prevalência não é apresentada apenas como um indicador epidemiológico, refletindo a doença no passado, mas composta por aspectos operacionais, tais como: capacidade de detecção de casos, duração do tratamento, taxa de cura e óbitos. Pela dependência de fatores operacionais, um reservatório de casos não detectado pode gerar uma predição menos confiável que as já realizadas por outros indicadores. Desta forma, apontam para a não existência de parâmetros estabelecidos para estimar prevalência oculta e referem-se aos métodos já apresentados como "adivinhos", porque apenas oferecem aproximações.

Em 1997, Andrade et al. ${ }^{10}$ verificaram significante correlação entre as variáveis operacionais do programa e aumento nos coeficientes de detecção, como melhoria das atividades de detecção da doença, tendo em vista a média nacional. No estudo desenvolvido por Motta e Zuniga ${ }^{6}$ observou-se uma tendência de crescimento da detecção da hanseníase no Brasil com diferenças regionais por meio do coeficiente de detecção, o que, naquela época, indicava um acúmulo de casos a serem detectados. Em 2002, Andrade e Velloso ${ }^{11}$ sugeriram importante magnitude da doença, especialmente nos
Estados da região Amazônica e em áreas de influência (incluindo o Estado de Mato Grosso), verificada principalmente devido à detecção de casos novos em menores de 15 anos, onde, para cada caso detectado em outras regiões do Brasil, em média, seis casos foram detectados na região Amazônica. Pode-se afirmar que, em Mato Grosso, mesmo com a implantação da PQT há mais de 10 anos, um reservatório de casos não detectado (prevalência oculta) mantém fontes de infecção da doença. Sabendo-se que o período de latência da hanseníase é longo e pouco conhecido, somado ao fato de novos indivíduos estarem sendo infectados na comunidade em conseqüência da insuficiente cobertura de PQT, espera-se um aumento na detecção de casos novos de hanseníase após uma intervenção de incentivo a esta atividade.

No método proposto por Gil Suárez e Lombardi ${ }^{3}$, apresentado com aparente facilidade de aplicação por se utilizar apenas de percentuais, constrói-se a prevalência por meio do registro de casos novos, neste caso sem se utilizar a base populacional. Em 2000, os autores reapresentaram o método como uma estratégia para a eliminação da hanseníase no sub-nível nacional, argumentando que a prevalência oculta de hanseníase seria influenciada por elementos epidemiológicos e operacionais, e que ambos podem ser avaliados usando-se a percentagem de casos novos detectados com alguma incapacidade física ${ }^{12}$. Assim como Ferreira et. al. ${ }^{4}$, baseiam-se em registros e avaliação de incapacidade física que dependem diretamente da qualidade da avaliação do grau de incapacidade física e da qualidade da informação gerada a partir desta variável.

Ferreira et al. ${ }^{4}$, na tentativa de elaboração de um método mais acurado, comentam sobre a arbitrariedade na decisão de escolha de 5 anos de atraso no diagnóstico de todos os casos com alguma incapacidade e tempo zero para aqueles que não desenvolveram incapacidades. A partir desta crítica, introduziram como parâmetro no cálculo de estimativa da prevalência oculta a variável tempo de atraso no diagnóstico, com 
base na data do início dos sintomas e data do diagnóstico registrado nos prontuário, além de utilizarem as categorias de comparação sexo, idade, classificação operacional e especialidade médica responsável pelo diagnóstico. O método proposto por Ferreira et al. ${ }^{4}$, como todos os outros, não leva em consideração o registro ativo, mas os casos detectados. Entretanto, os casos identificados como prevalência oculta devem ser somados às taxas de prevalência, neste caso, um indicador epidemiológico calculado a partir do registro ativo e com base populacional $^{13}$. Utiliza-se a relação que, para cada caso detectado com incapacidade física, existiria outro caso não detectado na comunidade alvo do estudo no período de cada ano de atraso no diagnóstico, sem ponderar o fato de existirem casos não bacilíferos com incapacidades físicas e que, portanto, não poderiam estar relacionados a novos casos. Assim, esta pode ser entendida com uma nova medida arbitrária. Afinal, quem pode afirmar quantos casos de hanseníase podem ser gerados ao ano a partir de um caso não detectado?

Outra dificuldade está na interpretação de cobertura do programa, sendo esta uma informação fundamental para a aplicação do método. Verificamos ainda que, pela atual base de dados do SINAN (versão Windows a partir de 2001), a variável data do início dos sintomas é substituída automaticamente pela variável data do diagnóstico. Desta forma, este método só pode ser aplicado se forem utilizadas bases de dados anteriores a 2001 ou por meio de informações coletadas em entrevistas com os indivíduos em tratamento. Nesse estudo foram utilizados os resultados obtidos pelos autores na década de setenta e oitenta, no Estado do Rio Grande do Sul, em que pessoas-tempo passam a ser casos ocultos, por meio da razão dos casos com incapacidade física, sendo uma pessoa ano equivalente a um caso oculto.

$O$ fato de terem sido utilizados o tempo entre o início dos sintomas e a data do diagnóstico do Estado do Rio Grande do Sul para o Estado do Mato Grosso poderia superestimar a prevalência oculta, considerando-se que nos últimos anos tenha havido aumento da acessibilidade ao diagnóstico, tendo em vista o aumento do número de unidades de saúde com alguma ação no controle da hanseníase nos últimos anos em Mato Grosso. Somam-se ainda as facilidades terapêuticas estabelecidas após a introdução da PQT. Por outro lado, sabe-se que continua havendo atraso diagnóstico pelas características dos casos detectados, especialmente pelo grau de incapacidade no momento do diagnóstico.

Quanto aos resultados obtidos por meio de tendência de crescimento na detecção da endemia, sugere-se que esta seja calculada por meio da média de casos estimados e não apenas pelo número de casos do último ano da série, que estaria mais suscetível às fragilidades operacionais dos serviços de saúde e da base de dados. Sem dúvida, espera-se que uma intervenção mobilizadora de profissionais de saúde aumente a capacidade de detecção. O problema é a definição do limite desta tendência em áreas de hiperendemicidade, como o Estado de Mato Grosso. Outro aspecto a ser considerado é que o uso constante deste método sempre eleva a estimativa, pois acrescenta sempre um percentual à detecção do ano anterior. Neste caso, não considera a possibilidade de redução da detecção ou esgotamento de casos ocultos.

Observando os resultados da estimativa da SES utilizada como meta de detecção, verificou-se, também neste caso, certa arbitrariedade na definição do número de casos novos. Possivelmente, a SES utilizou dados não padronizadas e informais como medida decisória, como, por exemplo, fatores operacionais e elementos subjetivos não disponíveis nas bases de dados, o que tornou o método não replicável. A partir dos resultados obtidos por meio da exclusão do nível endêmico, pode-se inferir que os municípios trabalharam vinculando a capacidade de detecção ao número de casos definidos enquanto meta, e não na busca da prevalência real da endemia.

Outro fato relevante é o pagamento de bônus vinculado à meta, podendo levar ao aumento do risco de detecção de casos falsos positivos ou "overdiagnosis". Contudo, 
neste caso não estamos modificando nossa análise em razão da possível presença de casos falso positivos, tendo em vista a tendência ascendente dos coeficientes de detecção de hanseníase em Mato Grosso apresentada por meio da análise de séries históricas ${ }^{11}$, indicando manutenção da prevalência oculta, ainda que seus valores sejam desconhecidos.

Segundo a Organização Mundial de Saú$\mathrm{de}^{14}$ não existe método eficaz e de baixo custo que permita estimar com precisão a prevalência real da hanseníase. Entretanto, existem métodos indiretos para análise do esgotamento de casos ocultos. Lechat, Andrade e Velloso ${ }^{9,11}$ referem-se ao acompanhamento dos casos por meio de séries históricas do grau de incapacidade física no momento do diagnóstico em que a redução dos casos Grau I e II, segundo os parâmetros de classificação da OMS e MS ${ }^{13,14}$, indicariam melhoria na capacidade de detecção, ou seja, casos detectados com incapacidades físicas avançadas indicam a ocorrência de detecção tardia. Assim, uma aproximação da avaliação da taxa de incidência consiste em calcular, por meio dos casos detectados, a proporção de pacientes com deformidades Graus I e II que deverá tender a zero. Verifica-se que, a partir dos casos novos detectados, pode-se analisar a capacidade operacional de detecção precoce ou o quanto o coeficiente de detecção se aproxima da incidência e não da prevalência. Variáveis como idade, combinadas com informações não apenas sobre incapacidades físicas, sexo, classificação operacional, devem ser somadas a informações sobre o número e o tipo de lesões no momento do diagnóstico. Outra possibilidade é a combinação de tempo e espaço dos casos de hanseníase, buscando identificar focos da doença ou áreas de risco.

Portanto, enquanto mecanismo de mobilização, a estimativa de casos novos de hanseníase mostra alguma efetividade na detecção de casos novos. Contudo, tal estimativa não pode ser reconhecida, especialmente pelos gestores e profissionais de saúde, como a possibilidade de esvaziamento imediato de reservatórios de casos mantidos ao longo dos anos, mas como uma estratégia de motivação para o trabalho das equipes de saúde na busca de casos novos.

\section{Conclusão}

Embora muitos esforços venham ocorrendo na busca do conhecimento da real prevalência da hanseníase por meio da prevalência oculta, conclui-se que os métodos publicados apresentam fragilidades e apenas sugerem a presença de reservatórios de casos em áreas com detecção tardia. Operacionalmente, parece mais adequado basear-se em séries históricas segundo idade, classificação operacional, grau de incapacidade física e número de lesões no momento do diagnóstico para a identificação de áreas de risco e, conseqüentemente, eliminação da hanseníase.

\section{Referências}

1. Mato Grosso, Secretaria de Estado de Saúde. Decreto 2.648 de 12 de junho de 2001 e Portaria, 062/GAB/SES. Projeto Prioritário “Tolerância Zero: Mato Grosso sem hanseníase.

2. Rothman KJ; Greeenland S. Modern Epidemiology. 2a ed. Lippincott Willians \& Wilkins; 1998. p. 235-7.

3. Gil Suàrez RG, Lombardi, C. Estimado de prevalência de lepra. Hansenologia Internationalis 1997; 22(2): 27-31.

4. Ferreira J, Mengue SS, Wagner MB, Duncan BB. Estimating Hidden Prevalence in Hansen's Disease Through Diagnosis Delay and Grade of Disability at Time of Diagnosis. Int J Lepr 2000; 68(4): 464-73.
5. Brasil, Ministério da Saúde. Guia para implantar/ implementar as atividades de controle da hanseníase nos planos estaduais e municipais de saúde. Brasília, 1999.

6. Motta CP, Zuniga M. Time Trends of Hansen's Diseases in Brazil. Int J Lepr Other Mycobact Dis 1990; 58(3): 453-61.

7. Bechelli LM; Martinez Dominguez V. Proposed methods for estimating leprosy prevalence. Bull World Organ 1966; 34: 811-26. 
8. Opromolla DV, Nóbrega RC, Gonçalves NNS, Padovani SHP, Padovani CR, Gonçalves A. Estimativa da prevalência da hanseníase pela investigação em demanda inespecífica de agências de saúde. Rev Saúde Pública 1990; 24(3): 178-85.

9. Lechat MF, Vanderverken M, Declercq E, Misson CB. Analysis of trends in the occurence of leprosy. Wld Health Statistics Quarterly 1986; 39: 129-37.

10. Andrade VLG, Militão de Albuquerque MDF, Chagastelles Sabroza P. The importance of operational factors for the interpretation of indicators in the Hansen's disease endemic in Brazil. Acta Leprológica 1997; 10(3): 131-9.
11. Andrade VLG, Velloso AP. Hanseníase: curar para eliminar. O Estágio da Eliminação no Brasil ao final do Século XX. Porto Alegre: Edição das Autoras; 2002. p. 51-62.

12. Gil Suàrez RG, Lombardi C. Leprosy Elimination at Sub-National level. Leprosy Review 2000; 71:206-11.

13. Brasil, Ministério da Saúde. Guia para o controle da hanseníase Cadernos de Atenção Básica. №10. Secretaria de Políticas de Saúde. Departamento de Atenção Básica; 2002.

14. Organização Mundial da Saúde. Definição do caso de lepra. Bol Epidemiol 2002; 23. n2.

recebido em: 03/11/03

versão reformulada apresentada em: 02/04/04

aprovado em: 26/04/04 